\title{
OSTEOMYELITIS OF THE JAWS IN INFANCY TREATED WITH PENICILLIN
}

\author{
BY \\ N. M. JACOBY, M.D., M.R.C.P. \\ Registrar to the Children's Department, Guy's Hospital; Physician E.M.S. \\ and \\ L. SAGORIN, M.R.C.S., L.R.C.P., D.C.H. \\ Paediatric R.M.O., Pembury Hospital, E.M.S.
}

Osteomyelitis of the maxilla is a rare condition, about eighty cases having been reported since 1847 . It produces a characteristic clinical picture, making recognition easy. Asherson (1939) in England and Lacy and Engel (1939) in the U.S.A. have given detailed accounts of the clinical picture and the differential diagnosis and have discussed the etiology and reviewed the literature. Their views on the treatment and prognosis agree fairly closely, but as these views antedate the use of penicillin, they are no longer tenable.

The condition is practically confined to infants under the age of twelve weeks, whose previous history in relation to delivery and feeding is not significant. The condition has generally been progressing for several days before it is recognized as being one of more than mere local inflammation. In the present cases, this period averaged eight days. The characteristic feature is gross swelling of the affected side. In the case of the maxilla both eyelids are involved with proptosis and chemosis; the cheek is remarkably swollen and indurated. In addition there is frequently a unilateral purulent nasal discharge and occasionally pus discharges externally from below the inner canthus of the eye. Inside the mouth the alveolus and the hard palate are swollen and inflamed, and sinuses may be discharging either from the alveolus or the bucco-gingival fold; the bucco-gingival space may be markedly narrowed. When the mandible is involved the swelling is mainly in the submental region, whilst inside the mouth, although the lower jaw is affected, the condition is essentially the same. From any of the purulent discharges, staphylococcus aureus can be cultured.

The etiology of this condition is not completely explained, since the portal of entry of the staphylococcus is unknown. Asherson (1939), Lacy and Engel (1939) and Mowlem (1944) agree that the bone infection is of haematogenous origin, though there is no record of a positive blood culture in their cases. Unfortunately in the first two cases here described this investigation was also omitted, but in the one case in which it was done a heavy growth of staphylococcus aureus was obtained within twentyfour hours. In the case of the maxilla, the bone infection is, on anatomical grounds, presumed to start in the tooth socket of the first molar. In mandibular infections the theories that have been advanced are 'diminution of local immunity' and 'specific organismal infection,' but as Mowlem points out these theories are 'meaningless and incapable of proof.' Although the tooth buds are not in close proximity to the vessels it is possible that the infection settles in a tooth socket.

\section{Case reports}

Case 1. E. M. C., a male, aged twenty-four days. The infant was full term, and born by normal delivery, weighing $10 \mathrm{lb}$. $4 \mathrm{oz}$. at birth. He was breast fed, and was admitted on 9.8.44. At the age of fourteen days the mother noted swelling of the right upper gum, and the following day considerable swelling of the right face. At sixteen days pus was discharging from three sinuses in the gum.

On admission the infant was extremely ill, with temperature $102^{\circ} \mathrm{F}$., pulse 130 , and respiration 30 per minute. The right face was extremely swollen, and was indurated and red. Both eyelids were swollen, occluding the palpebral fissure; there was a discharge due to purulent conjunctivitis. The right side of the hard palate and upper alveolus were swollen and indurated. Pus was discharging from three sinuses on the alveolar margin.

TREATMENT. 9.8.44. OPERATION. Under general anaesthesia the right maxilla was opened through an incision of the gum. The sinuses were connected and about two drachms of pus was evacuated together with two small sequestra and four deciduous tooth buds. The resulting cavity was packed with vaseline gauze which was removed next day. (Culture from the pus gave a mixed growth of staphylococcus aureus and a few colonies of streptococcus haemolyticus. Both organisms were penicillin-sensitive.)

Post-operatively, intra-muscular penicillin 5000 units, was injected four-hourly. 
11.8.44. Intramuscular penicillin 2500 units four-hourly, was given until 18.8.44, the total dose being 160,000 units in nine days.

The temperature subsided to $98 \cdot 4^{\circ} \mathrm{F}$. in twentyfour hours and did not rise again; the infant was obviously out of danger from this time on. The facial swelling did not subside appreciably and by 14.8.44 there were fluctuant swellings over the right maxilla and below the inner canthus of the right eye.

15.8.44. $1 \mathrm{oz}$. pus was aspirated from within the mouth to drain the maxillary swelling. Pus below the inner canthus was aspirated from the skin surface.

20.8.44. The face was now completely symmetrical, and all swelling had subsided.

24.8.44. Discharged from hospital. The infant was last seen in May 1945 and has remained well.

Case 2. A.J.B., male, aged ten weeks. The infant was born at full term by normal delivery, weighing $7 \mathrm{lb} .13 \mathrm{oz}$. and was breast fed.

1.1.45. The mother noticed a small boil below the chin.

10.1.45. Submental swelling was present over the whole of the left side of the lower jaw.

14.1.45. Pus was discharging into the mouth.

15.1.45. Patient was admitted to hospital. The infant was not acutely ill, the temperature being $100^{\circ} \mathrm{F}$., the pulse 140 , and the respiration 44 per minute. There was a large, red, indurated swelling under the left inferior margin of the mandible. The left lower alveolus and floor of mouth were swollen. Three sinuses were discharging from the alveolus. X-ray showed irregularity of the lower mandibular border.

TREATMENT. 15.1.45. Intramuscular penicillin, 7000 units four-hourly, was given until 22.1 .45 , the total dose being 280,000 units.

The temperature fell within twenty-four hours and within seven days all swelling and discharge had ceased.

30.1.45. Prophylactically, a second course of penicillin was given (175,000 units in three days).

6.2.45. Patient was discharged fit from hospital.

July 1945. The private doctor reports that the child has remained well.

Case 3. B. W., a male, aged six weeks, had been born two weeks prematurely of a diabetic mother after surgical induction. The delivery was normal, and the birth weight $8 \mathrm{lb}$. $9 \mathrm{oz}$. The infant was artificially fed on a full cream feed.

26.6.45. The mother noted that the left eye was puffy and the left side of the face swollen. There was a watery discharge from the eye, and some difficulty in breathing.

27.6.45. The swelling had increased, and there was a purulent (later blood-stained) discharge from the left nostril.

28.6.45. The patient was admitted to hospital. The infant was not acutely ill, the temperature being $103^{\circ} \mathrm{F}$., the pulse 140 , and the respiration 40 per minute. The left side of the face and the eye-lids were oedematous. There was a large, red, indurated, tender area over the left maxilla and a purulent discharge from the left nostril and eye. The nasal discharge increased on pressure over the maxilla. The left alveolus and hard palate were swollen and buccogingival space was narrowed but there was no sinus apparent.
Blood culture gave a growth of staphylococcus aureus, and a culture of the nasal discharge grew a similar organism which was penicillin-sensitive.

TreatMENT. 28.6.45. Intramuscular penicillin, 5000 units four-hourly, was given until 6.7.45, the total dose being 235,000 units.

29.6.45. A small sinus discharged in the posterior part of the left upper alveolus; a probe was inserted about $\frac{1}{8}$ inch.

The temperature fell to $98.4^{\circ} \mathrm{F}$. in twenty-four hours and did not rise again. The swelling was subsiding by 30.6 .45 and both this and the nasal discharge had completely disappeared by 6.7 .45 , when the sinus too was closed.

11.7.45. The patient was discharged from hospital. The infant was last seen on 1.8.45 and had remained well.

\section{Comment}

TREATMENT. In case 1 no previous experience of the use of penicillin in this condition was available. In retrospect it now appears that the surgery undertaken was far too radical and without such interference there would probably have been a smaller loss of deciduous teeth. Subsequent experience has taught us that the best procedure is to rely mainly on penicillin and to carry out only such surgical measures as may be necessary to remove obvious collections of pus. Thus whilst in case 1 an operation was performed first and penicillin used afterwards, in the subsequent cases penicillin was used from the very beginning and it was found that no surgery was required.

In case 2 where the mandible was involved, a second prophylactic course of penicillin was given one week after the termination of the first, as in view of the recurrences reported in other cases of osteomyelitis it was thought that a second course might help to prevent such a recurrence here. In the maxillary cases, response to initial treatment was excellent and considering the vascular nature of this bone in infancy, we did not think it necessary to repeat the penicillin.

Prognosis. Penicillin therapy which has revolutionized the prognosis in so many infections, has apparently an equally dramatic effect in osteomyelitis of the jaws in infancy. Moreover, when dealing with infections of the face, not only must the threat to life be considered, but in those who survive the cosmetic result is of paramount importance.

In the past when treatment has consisted of free surgical drainage and more recently with the addition of sulphonamides, the mortality in 43 cases reported or collected by Marx (1922), Turner (1942), Magnus (1944), Asherson (1939) and Lacy and Engel (1939) was 31 per cent. In our three cases there were no deaths and though this series is too small to draw any definite conclusions, the fact that within twenty-four hours of starting treatment it was obvious that all the cases were going to recover and all danger had passed, leads us to deduce that in the future the mortality rate will be of a very low order.

The cosmetic result, judging from photographs of published cases treated by surgical drainage, has not 
been perfect. There are usually one or more scars below the lower eye-lid and these scars appear to be fixed to bone with resulting 'pitting' of the area. In our first case in which pus was aspirated through the skin, scarring was minimal and there was no disfigurement. The subsequent cases had no scarring and the cosmetic result was perfect.

The loss of tooth buds is a further factor that should be mentioned, as it may lead to deformity and disfigurement when the teeth erupt later on. $\mathrm{Rix}$, in a private communication, informs us that loss of tooth buds in the upper jaw is not likely to result in deformity, but when tooth buds are lost from the lower jaw the loss is more serious, as the shape of the upper dental arch appears to be controlled by the shape of the lower arch. As the loss of tooth buds is in part due to surgical intervention it is obvious that when penicillin is used and surgery is minimal, deformity due to loss of teeth of the primary dentition will also be minimal.

A last point worthy of mention is the remarkable rapidity with which penicillin cures the disease. Thus, whereas the average duration of the surgically treated cases is about three and a half months, and even after this period some of them still had discharging sinuses, our three penicillin-treated cases were completely cured and all sinuses healed in twelve days.

\section{Summary}

(1) The penicillin treatment of two cases of osteomyelitis of the maxilla and one of the mandible, in infancy, is described.

(2) The prognosis and cosmetic result are dramatically improved and the duration of the disease greatly reduced.

Thanks are due to Dr. A. W. Taylor for assistance with the penicillin dosage, and Mr. E. C. Hughes and Mr. J. J. Bell for their surgical advice.

\section{REFERENCES}

Asherson, N. (1939). J. Laryng., 54, 691.

Lacy, N. E., and Engel, L. P. (1939). Arch. Otolaryng., Chicago, 29, 417.

Magnus, J. A. (1944). Brit. J. Ophthal., 28, 135.

Marx, E. (1922). Ibid., 6, 25.

Mowlem, R. (1944). Brit. med. J., 1, 517.

Turner, J. H. (1942). Arch. Pediat., 59, 1. 